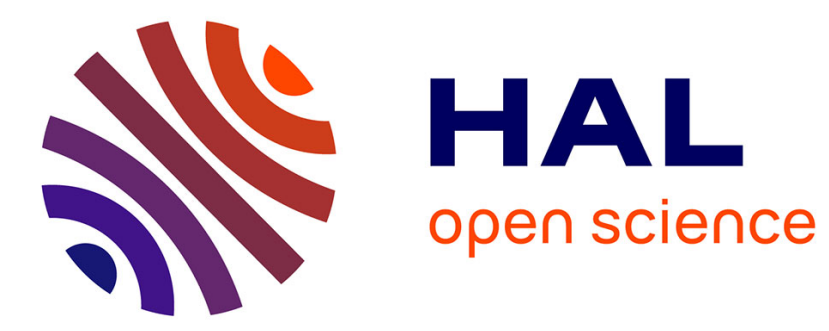

\title{
A framework for optimal decentralized power control with partial CSI
}

Achal Agrawal, Samson Lasaulce, Olivier Beaude, Raphael Visoz

\section{To cite this version:}

Achal Agrawal, Samson Lasaulce, Olivier Beaude, Raphael Visoz. A framework for optimal decentralized power control with partial CSI. Fifth International Conference on Communications and Networking (ComNet'2015), Nov 2015, Hammamet, Tunisia. pp.1 - 7, 10.1109/COMNET.2015.7566628 . hal-01437588

\section{HAL Id: hal-01437588 \\ https://hal.science/hal-01437588}

Submitted on 17 Jan 2017

HAL is a multi-disciplinary open access archive for the deposit and dissemination of scientific research documents, whether they are published or not. The documents may come from teaching and research institutions in France or abroad, or from public or private research centers.
L'archive ouverte pluridisciplinaire HAL, est destinée au dépôt et à la diffusion de documents scientifiques de niveau recherche, publiés ou non, émanant des établissements d'enseignement et de recherche français ou étrangers, des laboratoires publics ou privés. 


\title{
A Framework for Optimal Decentralized Power Control with Partial CSI
}

\author{
Achal Agrawal ${ }^{1}$, Samson Lasaulce ${ }^{1}$, Olivier Beaude ${ }^{1}$ and Raphael Visoz ${ }^{2}$ \\ ${ }^{1}$ L2S (CNRS - CentraleSupélec- Univ. Paris Sud 11), France \\ ${ }^{2}$ Orange Labs, Issy-les-Moulineux, France \\ ${ }^{1}$ \{agrawal, lasaulce, beaude\}@1ss.supelec.fr, ${ }^{2}$ raphael.visoz@orange-ftgroup.com
}

\begin{abstract}
In this paper, we use a recent information theoretical result to develop a general framework for finding optimal power control policies in the case of interference channels. The aforementioned result characterizes the achievable payoffs for an $N$-Agent (transmitters in our application) coordination problem with a certain information structure. We then provide an algorithm which exploits the characterization of achievable payoffs by conditional probability distributions to find optimal decision functions for the transmitters. Due to its general nature, the developed framework is conducive for applications to diverse scenarios in wireless communications. In this article, we restrict our attention to the case of decentralized power control in interference channels for different utility functions namely sum-rate, sum-energy and sum-goodput. Our approach has the following salient features: 1) The method proposes optimal power control functions for any given utility function as opposed to ad-hoc solutions for different utilities proposed in the literature, and 2) Noise in the channel estimation is taken into account, thus providing robust optimal solutions.
\end{abstract}

\section{INTRODUCTION}

One of the key contributions of this paper is to exploit the recent theorem derived in [1] to find power control functions which may exploit the available knowledge optimally (in the long run). As an example, we treat the well studied problem of optimal power control in interference channels ([2], [3], [4]) and show that not only does our framework attain various payoffs identical to the state of the art, it also provides the optimal power control functions in diverse scenarios. The scenarios considered resemble those of [5], [6]. These works propose ad hoc solutions for specific utilities but not a generic framework which directly provides a power control function that aims at exploiting the available and arbitrary information as well as possible. Indeed our approach is very general and can be used to treat more complex and interesting scenarios by considering different kinds of channel state informations available at transmitters, different common objectives, robust power control taking the noisy communications into account, vectorial optimization etc.

The generality of the approach is by virtue of exploitation of some recent information theoretical results [1]. A similar approach, albeit with a different information structure and for only 2 transmitter-reciever pairs was proposed in [7]. The information structure considered in this paper is more adapted for the application proposed under realistic assumptions, the important assumptions being 1) No knowledge of future realizations of the nature state w.r.t. which the agents are coordinating, 2) Any number of agents with only a partial and noisy information about the current nature state, and 3) Decision functions must be found in a decentralized manner for having more robustness leading to simpler design architectures.

Indeed, for the case of power control in wireless interference channels, these assumptions have been paid some attention. The necessity of minimizing the information required at the transmitters for coordination led to approaches proposed in [8]), [9], [10]. They consider only local channel state information at the transmitters (CSIT). This is also important for reducing the complexity of the optimizations to be performed. For sum-rate maximization, the scenario considered by us is comparable to a single-carrier version of the iterative water filling algorithm (IWFA) [11]. In the case of sum-energy utility, channel inversion strategy was proposed by [12], whereas the multi-carrier version of the problem was considered by [13]. Decentralization of the power control, or for that matter for any wireless network design, is the reason why game theory has been extensively applied to such problems [14]. We thus compare our results to game theoretic equilibria analysis as most of the decentralized optimization literature only guarantees convergence to Nash equilibria.

The article follows the structure from more general results to specific application of the framework proposed. To this effect, Sec. II describes the performance limits of a general coordination problem characterizing the optimal achievable payoffs. The characterization lays the foundation for an algorithm proposed in Sec. III for finding the optimal decision policies of the coordinating transmitters. While we continuously illustrate what the developed framework corresponds to for wireless applications, in Sec. IV-A we describe the specific problem of power control in interference channels. A numerical analysis is conducted for diverse scenarios and the results are compared to existing literature in Sec. IV-B. Concluding remarks are provided in $\mathrm{V}$

\section{Performance limits of an $N$-Transmitters INTERFERENCE COORDINATION PROBLEM}

Consider a coordination problem where $N$ transmitterreceiver pairs are trying to coordinate the power emitted by them $X_{i} \in \mathcal{X}_{i}, i \in\{1, \ldots, N\}$ with respect to a nature state represented by $X_{0} \in \mathcal{X}_{0}$ to optimize a common payoff function $w\left(x_{0}, x_{1}, x_{2}\right)$ over a long time period $T$. The nature state $X_{0}$ for applications to wireless communications is the channel gain matrix $g_{i j}=\left|h_{i j}\right|^{2}$, with $g_{i j}$ being the 
channel gain of the link between transmitter $i$ and reciever j. $X_{0}=\left(g_{11}, \ldots, g_{N N}\right)$ is a random state which affects the common payoff function for the system and is not controlled by the coordinating transmitters. The realizations of the nature state $X_{0, t}$ at each time instant $t$ are i.i.d. and follow a distribution $\rho$. In wireless communications $\rho$ is typically an exponential distribution for each channel gain $g_{i j}$. Our aim is to characterize all the achievable expected payoffs under a certain information structure over the large time period $T$, $T \rightarrow \infty$.

Firstly, we need to define the information structure under consideration. At every instant $t$, all the transmitters are assumed to have an image or a partial observation $S_{i, t}$ of the nature state $X_{0, t}$ with respect to which they are coordinating. In the case of our application, this could be knowledge of only local CSIT, i.e. transmitter $i$ observes a noisy version (in general) of only the direct link channel gain $g_{i i}$. One could imagine other kinds of information available at the transmitters: for example, transmitter $i$ observes all the links $g_{j i} \forall j$. The observations $S_{i, t}$ are assumed to be generated by a memoryless channel whose transition probability is denoted by $\urcorner_{i}\left(S_{i, t} \mid X_{0, t}\right)$. All transmitters have to choose the power emitted by them $X_{i, t}$ based on this information received. Formally, the sequences of decision functions for each agent $i, \sigma_{i, t}$ is defined as:

$$
\begin{array}{rlrl}
\sigma_{i, t}: & \mathcal{S}_{i}^{t} \times \mathcal{U} & \rightarrow \mathcal{X}_{i} \\
\left(s_{i}(1), s_{i}(2) \ldots s_{i}(t), u(t)\right) & \mapsto x_{i}(t)
\end{array}
$$

where $\mathcal{S}_{i}^{t}=\mathcal{S}_{i}(1) \times \mathcal{S}_{i}(2) \ldots \times \mathcal{S}_{i}(t)$ is the discrete observation alphabet till the instant $t$ and $\mathcal{X}_{i}$ is the power emitted for transmitter $i$ with $\left|\mathcal{S}_{i}\right|,\left|\mathcal{X}_{i}\right|<\infty$. $\mathcal{U}_{t}$ is the alphabet of the auxiliary variable $U$ which is discussed in more detail later. $s_{i}(t), u(t)$ and $x_{i}(t)$ are the realizations of the corresponding variables at instant $t$.

The problem is said to be decentralized as each transmitter chooses its power independently based on the information received by it. Since the channel gains are generated by a random process, and thus in general also the corresponding chosen power levels, the quantity to be optimized is the expected objective function:

$$
\mathbb{E}_{Q}[w(X)]=\sum_{x \in \mathcal{X}} w\left(x_{0}, x_{1}, \ldots, x_{N}\right) Q\left(x_{0}, x_{1}, \ldots, x_{N}\right)
$$

where $X=\left(X_{0}, X_{1}, \ldots, X_{N}\right), x=\left(x_{0}, x_{1}, \ldots, x_{N}\right)$, and $\mathcal{X}=\mathcal{X}_{0} \times \prod_{i=1}^{N} \mathcal{X}_{i}$. An important point to note is that since expectation is a linear operator, optimizing the expected payoff is equivalent to finding the optimal distribution $Q\left(x_{0}, x_{1}, \ldots, x_{N}\right)$. However, the optimization problem is not so trivial as indeed there are certain restrictions on the distributions $Q$ that are implementable given the imposed information structure. For doing that, one first needs to define the notion of an implementable distribution.

Definition 1 (Implementability). Let the information structure be as defined in (1). The probability distribution
$\bar{Q}\left(x_{0}, x_{1}, \ldots, x_{N}\right)$ is implementable if there exist decision functions $\sigma_{i, t}$ such that as $T \rightarrow+\infty$, we have for all $x \in \mathcal{X}$,

$$
\frac{1}{T} \sum_{t=1}^{T} Q_{X_{0} \ldots X_{N}, t}\left(x_{0}, \ldots, x_{N},\right) \rightarrow \bar{Q}\left(x_{0}, \ldots, x_{N}\right)
$$

where $Q_{X_{0} X_{1} \ldots X_{N}, t}=Q_{X_{1}, \ldots X_{N} \mid X_{0}, i} \times \rho$ is the joint distribution induced by $\sigma_{i, t}$ at stage $t$.

As seen before, the expected payoff is characterized by the probability distribution $Q$ over all the variables that intervene in the payoff function. Thus, the time averaged expected payoff $\bar{w}$ is said to be achievable, if and only if the corresponding distribution $\bar{Q}$ is implementable. The following theorem characterizes precisely the distributions that are implementable under the information structure (1).

Theorem 1. [1] Assume the random process $X_{0, t}$ to be i.i.d. following a probability distribution $\rho$ and the available information to the transmitters $S_{i, t}$ to be the output of a discrete memoryless channel obtained by marginalizing the conditional probability $\neg\left(s_{1}, \ldots, s_{N} \mid x_{0}\right)$. An expected payoff $\bar{w}$ is achievable in the limit $T \rightarrow \infty$ if and only if it can be written as:

$$
\begin{gathered}
\bar{w}=\sum_{x_{0}, x_{1}, \ldots x_{N}, u, s_{1}, \ldots s_{N}} \rho\left(x_{0}\right) P_{u}(u) \times \\
\urcorner\left(s_{1}, \ldots s_{N} \mid x_{0}\right) \times \\
\left(\prod_{i=1}^{N} P_{X_{i} \mid S_{i}, U}\left(x_{i} \mid s_{i}, u\right)\right) w\left(x_{0}, x_{1}, \ldots x_{N}\right)
\end{gathered}
$$

where $U$ is an auxiliary variable which can be optimized.

The auxiliary variable $U$ is an external lottery known to the transmitters beforehand which can be used to achieve better coordination. An example for how this variable $U$ can help coordinate better in the case of power control is shown in Sec. IV-A.

Theorem 1 helps us find the best achievable payoffs on an average with arbitrary information to the transmitters as long as it follows the structure (in terms of when the information is available to whom) defined in equation (1). However, this theorem does not provide optimal sequences of decision functions $\sigma_{i, t}$ for the time averaged expected payoff. In the following section, we shall give an algorithm which helps find a suboptimal solution for the decision functions. The suboptimality is due to the high complexity of a multilinear optimisation problem. Nonetheless, In Sec.IV-B we see that the solutions obtained using this algorithm match the state of the art solutions proposed for different utilities discussed.

\section{AlgORITHM FOR FINDING SUB-OPTIMAL LOW-COMPLEXITY DECISION FUNCTIONS}

As seen from Theorem 1, optimal performance for the coordination problem depends only on the vector of conditional probabilities $\left(P_{X_{1} \mid S_{1}, U}, \ldots, P_{X_{N} \mid S_{N}, U}\right)$. Thus, it suffices to find an optimum vector of lotteries for every action $X_{i}$ possible to obtain the best achievable payoff. However, one can see that this task is computationally very demanding. Instead, we apply a suboptimal approach by searching for 
the conditional probabilities in a distributed manner, thus reducing the complexity of the search. To this effect, we apply sequential best response dynamics [14]. This consists of each transmitter choosing the best corresponding power given that others keep their powers constant, and each transmitter doing so sequentially within an iteration. Note that by doing so, the optimization problem becomes linear, and hence its solution lies at a vertex, i.e. either $\left(P_{X_{i} \mid S_{1}, U}\right)=0$ or $\left(P_{X_{i} \mid S_{1}, U}\right)=1$. Thus the search for conditional probability distributions simplifies to search for decision functions

$$
\begin{array}{rll}
f_{i}: \mathcal{S}_{i} \times \mathcal{U} & \rightarrow \mathcal{X}_{i} \\
& \left(s_{i}(t), u(t)\right) & \mapsto x_{i}(t)
\end{array}
$$

An important point to note is that we search for stationary strategy, i.e. $f_{i}$ does not depend on the time instant $t$. This simplification is practical as it leads to simpler design structures and easier to find in terms of complexity. Also, the auxiliary variable $U$, which serves a coordination key, is exchanged offline beforehand and thus does not entail any signalling cost.

The procedure outlined below is performed many times till convergence is achieved, typically in a few iterations. The number of iterations required for convergence of course depends on the number of agents coordinating, but it scales up very slowly as best response dynamics normally converge very fast. Furthermore, since we are considering a common 'team' payoff $w\left(x_{0}, x_{1}, \ldots, x_{N}\right)$, the convergence of best response dynamics is guaranteed [15].

Proposition 1. Algorithm 1 converges always for a common performance criteria $w\left(x_{0}, x_{1}, \ldots, x_{N}\right)$.

Proof: The result can be proved by induction or more generally by calling for an exact potential game property (the latter argument may hold in the more general case in which the transmitter have different performance criteria).

For further justification of the simplification made by finding functions $f_{i}$ instead of conditional probability distributions, note that the optimization problem is linear w.r.t. each component of the vector $\left(P_{X_{i} \mid S_{1}, U}\right)$ that we are searching. The problem is therefore multilinear and Algo 1 will converge to the vertex of the polytop defined by the constraints. Note however that we might lose out on finding the global optimum by doing so. Nonetheless, the power control functions obtained by the simplification are sufficient to compare with the existing literature which typically provide optimal control functions and not mixed randomized strategies.

To apply best response dynamics, we rewrite the expected utility given by Theorem 1 in the following way:

$$
\begin{aligned}
\bar{w}= & \sum_{x_{0}, x_{1}, \ldots x_{N}, u, s_{1}, \ldots s_{N}} \rho\left(x_{0}\right) P_{U}(u) \times \\
& \neg\left(s_{1}, \ldots, s_{N} \mid x_{0}\right) \times \\
& \left(\prod_{i=1}^{N} P_{X_{i} \mid S_{i}, U}\left(x_{i} \mid s_{i}, u\right)\right) w\left(x_{0}, x_{1}, \ldots x_{N}\right) \\
= & \sum_{a_{i}, b_{i}, u} \theta_{a_{i}, b_{i}, u} P_{X_{i} \mid S_{i}, U}\left(x_{a_{i}} \mid s_{b_{i}}, u\right)
\end{aligned}
$$

where $a_{i}, b_{i}, u$ are the respective indices of $x_{i}, s_{i}, u$ and

$$
\begin{gathered}
\theta_{a_{i}, b_{i}, u} \triangleq\left[\sum_{a_{0}} \rho\left(x_{a_{0}}\right)\right\rceil_{i}\left(s_{i} \mid x_{a_{0}}\right) \sum_{a_{-i}} w\left(x_{a_{0}}, x_{a_{1}}, \ldots x_{a_{N}}\right) \times \\
\left.\left.\sum_{b_{-i}} \prod_{i^{\prime} \neq i}\right\rceil_{i^{\prime}}\left(s_{b_{i^{\prime}}} \mid x_{a_{0}}\right) \prod_{i^{\prime} \neq i} P_{X_{i^{\prime}} \mid S_{i^{\prime}}, U}\left(x_{i^{\prime}} \mid s_{b_{i^{\prime}}}, u\right)\right] P_{U}(u)
\end{gathered}
$$

where $a_{-i}, b_{-i}$ are the indices which represent $a_{i}, b_{i}$ being constant, while all the other indices are summed over. Written in this form, the linearity of the problem w.r.t. any one of the variables $P_{X_{i} \mid S_{i}, U}\left(x_{i} \mid s_{i}, u\right)$ is even more explicit. Note that we have also assumed the independence of the observation channels of all the agents, i.e. $\left.\neg\left(\underline{s} \mid x_{0}\right)=\neg_{1}\left(s_{1} \mid x_{0}\right) \times \ldots \times\right\urcorner_{N}\left(s_{N} \mid x_{0}\right)$. For interference channels, this assumption is reasonable as all transmitters receive independent feedback of the channel gains.

An important point to note is that the transmitters can run this algorithm offline as all they need to know are the channel statistics $\rho$. This helps in using the optimal functions for the entire timeslot where the channel statistics remain constant right from the start as opposed to taking some time initially to find the optimal functions online. The latter is the case for some decentralized solutions proposed such as the algorithms based on water-filling techniques proposed in [5], [6].

\section{Application to POWER CONTROL in INTERFERENCE CHANNELS}

In the previous sections, we developed the framework, giving intuition as to what the defined quantities might represent in diverse optimization problems of wireless communications. In this section, we concentrate on the problem of power control in interference channels [14] and provide numerical analysis of some special cases for the above problem.

\section{A. System Model}

Consider $\mathrm{N}$ single-antenna Transmitter-Receiver pairs Transmitter $_{\mathrm{i}}$, Reciever,$i \in\{1,2 \ldots, N\}$ with a single band interference channel with the channel gains being $g_{i j}, i, j \in$ $\{1,2 \ldots, N\}$ and $g_{i j} \in \mathcal{G} . \mathcal{G}$ is considered to be a discrete set and represents the alphabet of the possible channel gains. A well accepted model of statistics for the channel gains $g_{i j}=\left|h_{i j}\right|^{2}$ is Rayleigh fading. In this model, due to central limit theorem, the real and the imaginary components of $h_{i j}$ 


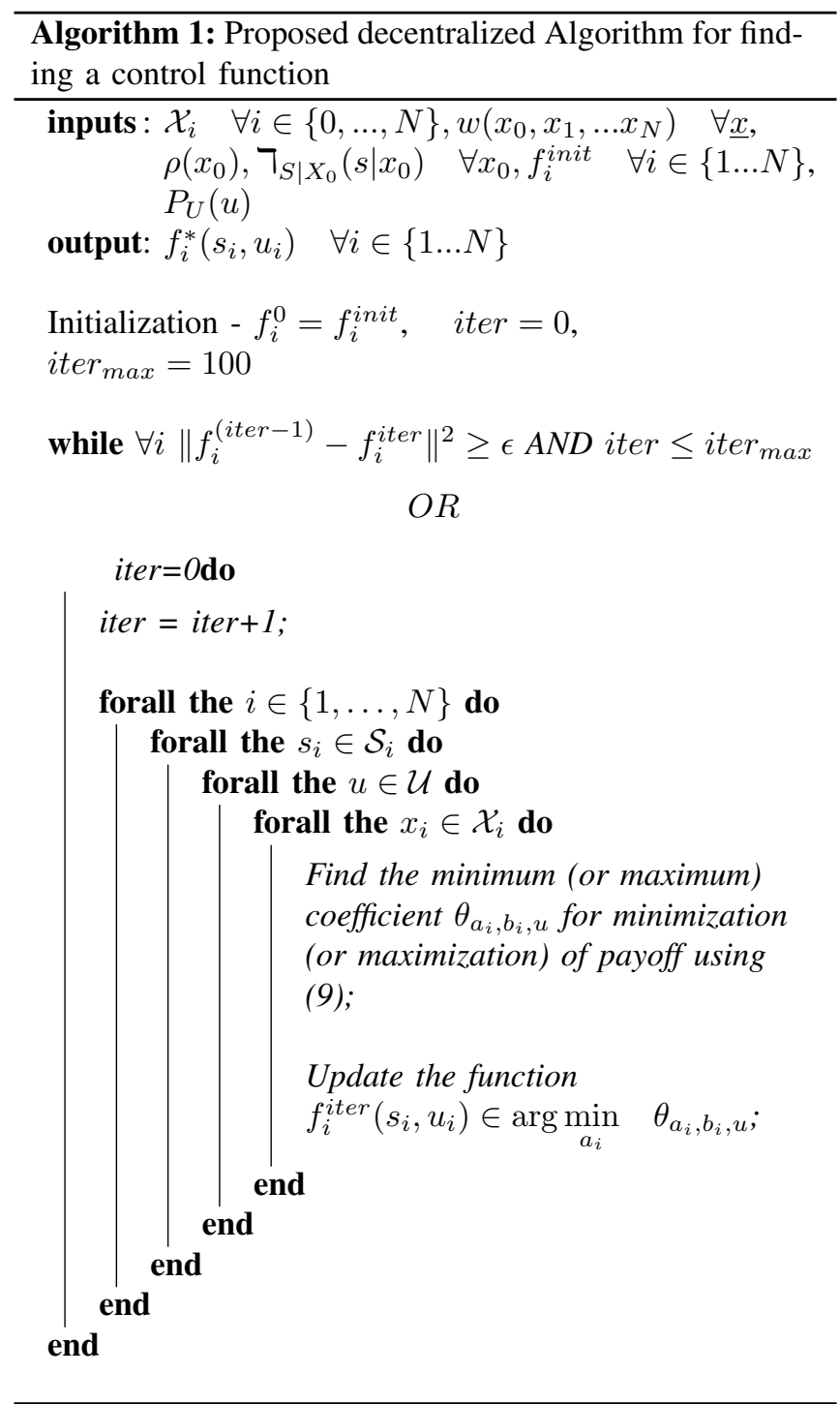

follow a normal distribution, and thus the channel gain $g_{i j}$ follows exponential distribution $\left(\left|h_{i j}\right|\right.$ follows Rayleigh distribution). We also assume that all channel gain distributions are independent of each other and that their realizations for each timeslot $t$ is i.i.d. The transmitters transmit at discrete power levels $P_{i} \in \mathcal{P}_{i}$ quantized uniformly (in $\mathrm{dB}$ ) between $\left[0, P_{\max }\right]$. The utility for the pair Transmitter ${ }_{i}$, Reciever ${ }_{i}$ depends on their signal to interference plus noise ratio (SINR) $\gamma_{i}(\underline{P}, \underline{g})$ where $\underline{P}$ is the vector with each component $P_{i}$ being the power emitted by the $i^{t h}$ transmitter. More precisely,

$$
\gamma_{i}(\underline{P}, \underline{g})=\frac{P_{i} g_{i i}}{\sigma^{2}+\sum_{i \neq j} P_{j} g_{j i}}
$$

where $\sigma^{2}$ is the noise variance. Without loss of generality, we'll consider $\sigma^{2}=1$, and change $P_{\max }$ to regulate the signal to noise ratio (SNR). SNR is defined to be the ratio $P_{\max } / \sigma^{2}$.
We shall consider only local CSIT, i.e. the direct channel gain $g_{i i}$ available at each transmitter $i$, based on which they have to choose the power level to emit at. For evaluation of the solutions found using our method, we shall consider 3 different payoff functions, which have vastly different optimal solutions and show that our method gives the same results as the state of the art which employ different methods for each payoff. The following common payoffs considered are:

- Sum-rate: $w_{R}(\underline{P}, \underline{g})=\sum_{i} \log \left(1+\gamma_{i}\right)$

- Sum-goodput: $w_{G}(\underline{P}, \underline{g})=\sum_{i} \Omega\left(\gamma_{i}\right)$

- Sum-energy: $w_{E}(\underline{P}, \underline{g})=\sum_{i} \frac{\Omega\left(\gamma_{i}\right)}{P_{i}}$

Typical functions for $\Omega$ are $\Omega(x)=e^{-\frac{c}{x}}$ [16], where $c>0$ or $\Omega(x)=\left(1-e^{-x}\right)^{M}$ where $M \geq 1$ is the packet length [12]. In simulations, we supposed $c=1$ as it only changes the optimal solutions by a multiplicative constant. Note that proposition 1 holds for the above payoffs as we are considering sum of individual payoffs which trivially satisfies the required condition for convergence.

The following table explicits the relation between the input variables for the algorithm 1 and the corresponding quantities for the application to power control defined above.

\begin{tabular}{l|l}
\hline General model & Power Control Application \\
\hline Nature State $-X_{0}$ & Channel state $-\left(g_{11}, \ldots, g_{N N}\right)$ \\
\hline Decision of Transmitter $i-X_{i}$ & Power emitted $P_{i} \in \mathcal{P}_{i}$ \\
\hline$w\left(x_{0}, x_{1} \ldots x_{N}\right)$ & $w_{R}(\underline{P}, \underline{g}), w_{G}(\underline{P}, \underline{g}), w_{E}(\underline{P}, \underline{g})$ \\
\hline$\rho\left(x_{0}\right)$ & $\prod_{i=1}^{N} \prod_{j=1}^{N} e^{-\bar{g}_{i j}}, \bar{g}_{i j}=\mathbb{E}\left(g_{i j}\right)$ \\
\hline$\neg\left(s_{i} \mid x_{0}\right)$ & $s_{i}=g_{i i}$ or $s_{i}=\widehat{g}_{i i}$ \\
\hline$f_{i}^{\text {init }}$ & $f_{i}=P_{\max } \forall s \in \mathcal{S}$ \\
\hline \multicolumn{2}{r}{ TABLE I }
\end{tabular}

CORRESPONDENCE BETWEEN THE GENERAL FRAMEWORK AND ITS APPLICATION TO POWER CONTROL

In Theorem 1, we introduced an auxiliary variable $U$ which could help in achieving better coordination. For better intuition, we show how a simply constructed external lottery helps transmitters coordinate in the case of power control.

Consider only $N=2$ transmitter-receiver pairs communicating over a single band interference channel while maximizing the sum-rate $w_{R}(\underline{P}, g)$. From [9], we know that the optimal solutions $\left(P_{1}^{*}, P_{2}^{*}\right) \in$ $\left\{\left(P_{\max }, 0\right),\left(0, P_{\max }\right),\left(P_{\max }, P_{\max }\right)\right\}$. However, if transmitters try to maximise their individual utility $\log \left(1+\gamma_{i}\right)$, the Nash equilibrium is $\left(P_{\max }, P_{\max }\right)$ [14].

Now consider the following probability distribution for a random variable $U$ with the realizations $u \in \mathcal{U},|\mathcal{U}|=3$.

$$
P_{U}(u)=\left\{\begin{array}{l}
\operatorname{Pr}\left(u=u_{1}\right)=\alpha_{1} \\
\operatorname{Pr}\left(u=u_{2}\right)=\alpha_{2} \\
\operatorname{Pr}\left(u=u_{3}\right)=\alpha_{3}=1-\left(\alpha_{1}+\alpha_{2}\right)
\end{array}\right.
$$

A large sequence of realizations for $U$ are drawn with the probability distribution specified in (11) and all transmitters 
share this drawn sequence. Now consider a trivial function common to all transmitters $\Delta: \mathcal{U} \rightarrow \underline{\mathcal{P}}$ which maps every realization of $U, u_{i}$ to one of three possible optimal power control vectors $\left\{\left(P_{\max }, 0\right),\left(0, P_{\max }\right)\left(P_{\max }, P_{\max }\right)\right\}$. Under this setup, one can do monte-carlo simulations for a large number of channel gain realizations, and find the optimal probabilities $\left(\alpha_{1}^{*}, \alpha_{2}^{*}\right)$ by doing an exhaustive search. This is done by searching over a discrete space $\alpha_{i} \in[0,1], i \in\{1,2$. For the discretization, the step size is chosen to be 0.01 for obtaining reasonably precise 'optimal' probability values.

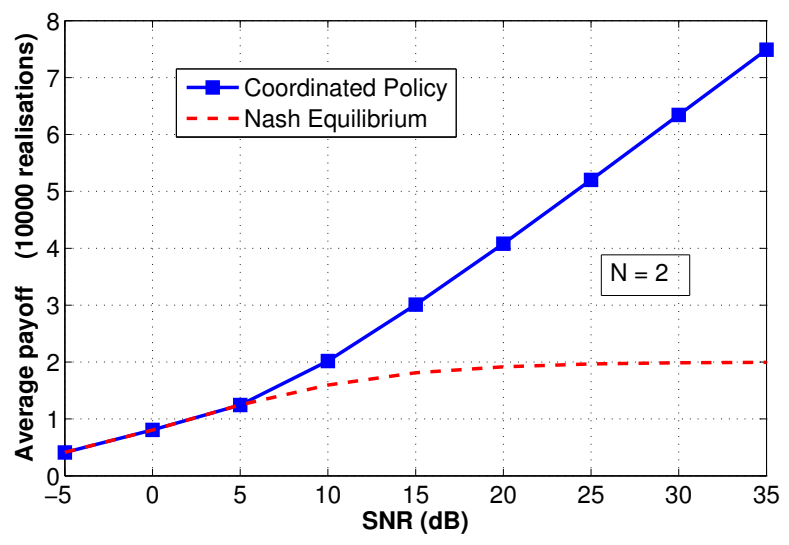

Fig. 1. The figure shows the performance in terms of sum-rate with : the proposed coordination mechanism using a public key shared beforehand; the single-carrier counterpart of the iterative water-filling algorithm. Without additional signalling, a significant gain is obtained.

In Fig. 1, we plot the average payoff for the coordinated policy using the pre-drawn lottery $U$ in terms of SNR (which is equal to $P_{\max }$ due to normalization of channel noise variance $\sigma^{2}$ ). We compare the average payoffs for the coordinated policy as well as for nash equilibrium and see that at higher SNR, it is indeed preferable to coordinate using $U$. This is not surprising at all, as at higher SNRs, the interference generated is too high, whereas time-sharing helps avoid this.

In the following section, we provide the simulation setup and discuss the power control functions given by our approach. We do not consider the pre-drawn lottery $U$ in our analysis, although in general $U$ will only help improve the coordination performance. This is because the solution where transmitters ignore the coordination key is always possible.

\section{B. Numerical Analysis}

1) Simulation Setup: We implement Algo. 1 for the case of power control in a single band interference channel [14]. To this effect, we use the correspondence established in Sec. IV-A. We consider the case when $N=2$. Note that exponential distributions for channel gain statistics $g_{i j}$ are characterized only by their mean $\bar{g}_{i j}$. We shall consider standard scenarios where $\left(\bar{g}_{i i}, \bar{g}_{i j}\right)=(1,0.3), i \neq j$.

As mentioned before, the noise variance of the channel has been normalized to one. Thus, the SNR is regulated via $P_{\max }=20 \mathrm{db}$. The possible power levels emitted by

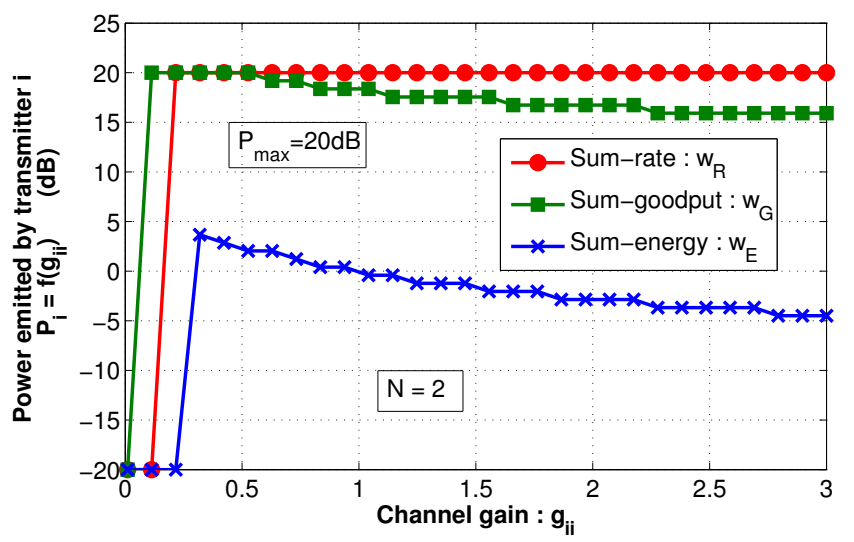

Fig. 2. The figure represents the power control functions provided by Algorithm 1 for the three performance metrics under consideration in this paper. In particular, it is seen that for maximizing sum-energy, the obtained power control function exhibits a threshold under which transmission should not occur for a given transmitter.

transmitters $\left(P_{i}\right)$ are uniformly quantized between $P_{i} \in$ $[-20,20]_{d b}$, with 50 quantization points.

Since the setup considered is symmetric for all Transmitter -Reciever pairs, we plot the power control functions $f_{i}\left(s_{i}\right)$, where $\mathcal{S}_{i}=\mathcal{G}_{i i}$, for the transmitter $i$. Note that since we do not consider the auxiliary variable $u$ from here on, the function $f_{i}$ is represented with only one argument $s_{i}$.

2) Results: In Fig. 2, we plot the power control functions $f_{i}$ obtained using the Algo 1 for the case $N=2$ against $g_{i i}$ the direct channel gain between the $i^{t h}$ Transmitter-Receiver pair. We do not consider noise in the channel estimation for the moment, and thus in this case $s_{i}=g_{i i}$. Three different utilities are considered, sum-rate, sum-energy, and sum-goodput.

For the case of sum-rate, it is known that binary power control $P_{i} \in\left\{P_{\min }, P_{\max }\right\}$ is optimal for 2 TransmitterReciever pairs [9]. Moreover, as shown in [8], optimal power control functions with only local CSIT $\left(g_{i i}\right)$ amounts to $P_{i}\left(g_{i i}\right)=P_{\min }$ if $g_{i i} \leq g^{*}$ and $P_{i}\left(g_{i i}\right)=P_{\max }$ otherwise.It is reassuring to find that our results verify this. Thus, in the case of sum-rate with local CSIT, we find exactly the same results as state of the art.

In the case of sum-energy, we see that all the available power $P_{\max }$ is not used. Indeed, even in the case of only 1 Transmitter-Reciever pair, the optimal power control function is $\frac{c}{g_{11}}$. We see that there is also a threshold value of $g_{i i}$ below which, $P_{i}=P_{\min }$. Above the threshold value, the function is similar to the optimal solution obtained in the case of only 1 Transmitter-Receiver pair. The threshold function is also seen as solution in the case of Sum packet rate. This is because the utility function is not monotonous w.r.t. $S I N R$. To the best of our knowledge, the power control functions found for sum-energy are new. Solutions in literature do not propose thresholding of power control functions to help reduce interference in case of bad direct channel gains. Admittedly, this requires greater analysis and comparisons with the state of the art. 


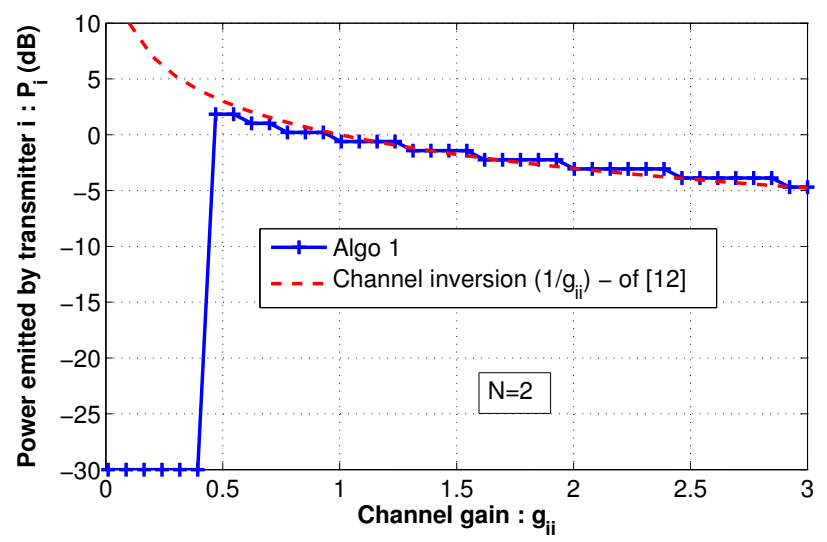

Fig. 3. Our algorithm reveals the shape of good power control functions in the presence of interference. In contrast with related works on energyefficiency, our work shows that thresholding is required to manage interference efficiently.

To investigate the function for sum-energy further, in 3 we compare the function obtained using our algorithm with that proposed by [12]. We see that unlike [12], we find a threshold below which emitting no power is more optimal. We also see that the presence of noise is makes the power control function more uniform. Our approach is shown to be much more robust to noise than one proposed by [12] as the function $1 / g_{i i}$ is very sensitive to noise at low values of $g_{i i}$.

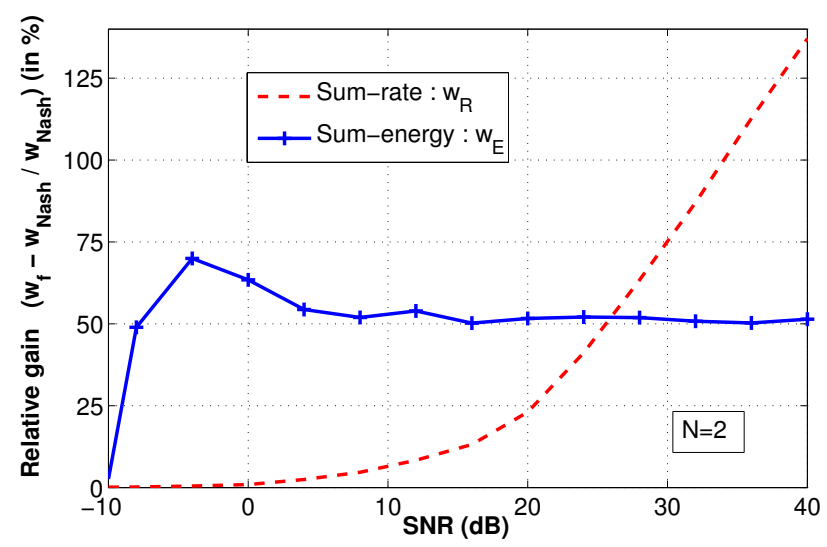

Fig. 4. Relative performance gain $\left(w_{f}-w_{N a s h} / w_{N a s h}\right.$ in \%) of our algorithm when compared to average payoffs obtained by Nash equilibrium for sum-rate and sum-energy. The curve for sum-energy saturates as at high SNR, $P_{\max }$ is not utilized as power emitted is much lower.

In Fig. 4, we compare the performance of the power control function with that of Nash equilibrium for different SNRs in the case of sum-energy and sum-rate. Thus, on the $y-a x i s$ we see the relative performance gain $\left(w_{f}-\right.$ $w_{\text {Nash }} / w_{\text {Nash }}$ in \% ) of our algorithm when compared to average payoffs obtained by Nash equilibrium. Current state of the art propose solutions which converge to the Nash equilibrium. Thus, the solutions are generally not optimal while maximizing common utilities that are sum of the individual utilities. The evaluations for obtaining average expected payoffs was done for $10^{6}$ channel realizations. They were drawn according to the distribution $\prod_{i=1}^{N} \prod_{j=1}^{N} e^{-\bar{g}_{i j}}$. The channel gain statistics $\bar{g}_{i j}$ were the same as the ones used for running the algorithm.

We see that for sum-energy, there is no performance gain at higher SNRs. This is because as seen from Fig. 2, the optimal power used is very low for all $g_{i i}$. However, we see that when compared to nash equilibrium, we obtain a significant gain of around $55 \%$. In the case of sumrate, performance obtained matches with [8] since the power control functions obtained are identical. Not suprisingly, at higher SNRs, Algo 1 does much better as Nash equilibrium, which is all transmitters transmitting at $P_{\max }$ generates too much interference.

One of the advantages of our framework is that it provides optimal power control functions even for noisy channel estimates. We illustrate this in Fig. 5 where power control functions for different levels of noise in channel estimation are plotted. This noise simulates the error in estimation due to noise in feedback transmission, or just simply statistical estimation error. The noise for the simulations is modeled to be gaussian, i.e. $s_{i}=g_{i i}+z$ where $Z \sim \mathbb{N}\left(0, \sigma_{z}^{2}\right)$. As expected, the power control functions become more uniform at higher noise levels, as the information received is less reliable, and thus transmitters emit at a power level which maximizes the utility for an average case.

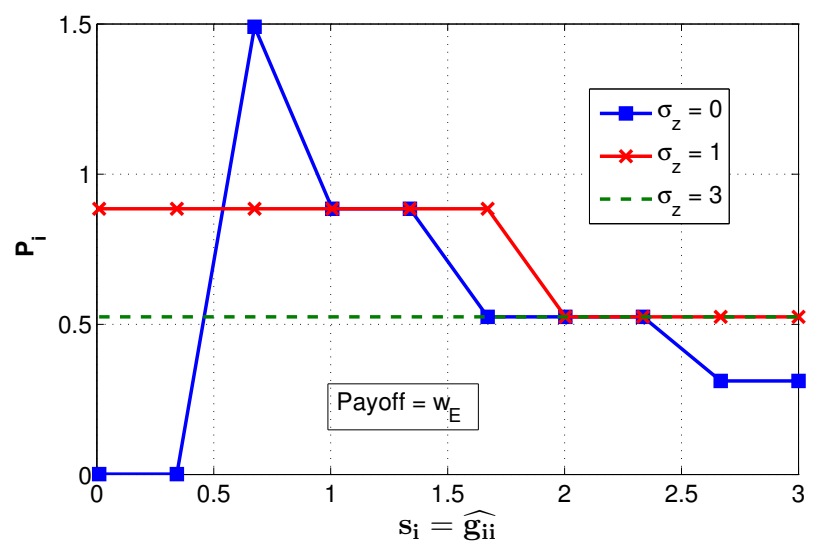

Fig. 5. Influence of channel estimation noise on the power control functions. We see that as noise increases, the optimal power emitted becomes more uniform. This is intuitive as higher the noise, higher the uncertainty in the observation leading to same power emitted for all channel gains observed in the asymptotic case.

\section{CONCLUSIONS}

The proposed framework was shown to be relevant in diverse scenarios of single band interference channel for finding optimal power control functions. Moreover, the power control functions depended only on local CSIT, thus having the merit of being implementable in a completely decentralized manner. Also, the solutions obtained take noise in the estimation of the channel gain into account. All the above features illustrate the generality of our approach in 
tackling problems of power control for maximizing sumutility functions.

However, the framework can be exploited further for tackling other problems in wireless communications as well. For example, one could consider the problem of power allocation in a multi-band interference channel. Also, the auxiliary variable $U$ was not exploited, which in general will only make the solution more optimal. One could also consider different information available at transmitter $i: g_{i j} \forall j$. In future, we hope to analyze more complex problems and show the merit of the proposed framework in finding decentralized strategies for transmitters to cooperate for optimizing common utilities.

\section{ACKNOWLEDGMENT}

Authors of this paper and particularly A. Agrawal would like to thank project LIMICOS - ANR-12-BS03-0005 for financing the project.

\section{REFERENCES}

[1] B. Larrousse, S. Lasaulce, and M. Wigger, "Coordinating partiallyinformed agents over state-dependent networks," in Information Theory Workshop (ITW), 2015 IEEE, April 2015, pp. 1-5.

[2] J. Zander, "Performance of optimum transmitter power control in cellular radio systems," Vehicular Technology, IEEE Transactions on, vol. 41, no. 1, pp. 57-62, 1992.

[3] R. Yates, "A framework for uplink power control in cellular radio systems," Selected Areas in Communications, IEEE Journal on, vol. 13, no. 7, pp. 1341-1347, Sep 1995.

[4] S. Stanczak, M. Wiczanowski, and H. Boche, Fundamentals of resource allocation in wireless networks: theory and algorithms. Springer Science \& Business Media, 2009, vol. 3.

[5] R. Gohary and T. Willink, "Robust IWFA for open-spectrum communications," Signal Processing, IEEE Transactions on, vol. 57, no. 12, pp. 4964-4970, Dec 2009.

[6] A. Anandkumar, A. Anandkumar, S. Lambotharan, and J. Chambers, "Robust rate maximization game under bounded channel uncertainty," Vehicular Technology, IEEE Transactions on, vol. 60, no. 9, pp. 44714486, Nov 2011.

[7] B. Larrousse, A. Agrawal, and S. Lasaulce, "Implicit coordination in 2-agent team problems. application to distributed power allocation," in IEEE 12th Intl. Symposium on Modeling and Optimization in Mobile, Ad Hoc, and Wireless Networks (WiOpt), May 2014.

[8] D. G. Paul de Kerret., Samson Lasaulce and U. Salim, "Best-response team power control for the interference channel with local CSI," in IEEE International Conference on Communications (ICC), June 2015.

[9] A. Gjendemsjø, G. E. Øien, and D. Gesbert, "Binary power control for multi-cell capacity maximization," in Signal Processing Advances in Wireless Communications, 2007. SPAWC 2007. IEEE 8th Workshop on. IEEE, 2007, pp. 1-5.

[10] A. Gjendemsj, D. Gesbert, G. Oien, and S. Kiani, "Binary power control for sum rate maximization over multiple interfering links," Wireless Communications, IEEE Transactions on, vol. 7, no. 8, pp. 3164-3173, August 2008 .

[11] G. Scutari, D. Palomar, and S. Barbarossa, "Asynchronous iterative water-filling for gaussian frequency-selective interference channels," Information Theory, IEEE Transactions on, vol. 54, no. 7, pp. 28682878, July 2008.

[12] D. Goodman and N. Mandayam, "Power control for wireless data," Personal Communications, IEEE, vol. 7, no. 2, pp. 48-54, Apr 2000.

[13] F. Meshkati, M. Chiang, H. Poor, and S. Schwartz, "A game-theoretic approach to energy-efficient power control in multicarrier CDMA systems," Selected Areas in Communications, IEEE Journal on, vol. 24, no. 6, pp. 1115-1129, June 2006.

[14] S. Lasaulce and H. Tembine, Game theory and learning for wireless networks: fundamentals and applications. Academic Press, 2011.

[15] D. Monderer and L. S. Shapley, "Potential Games," Games and Economic Behavior, vol. 14, no. 1, pp. 124-143, 1996. [Online]. Available: http://www.sciencedirect.com/science/article/pii/S0899825696900445
[16] E. V. Belmega and S. Lasaulce, "Energy-efficient precoding for multiple-antenna terminals," Signal Processing, IEEE Transactions on, vol. 59, no. 1, pp. 329-340, 2011. 